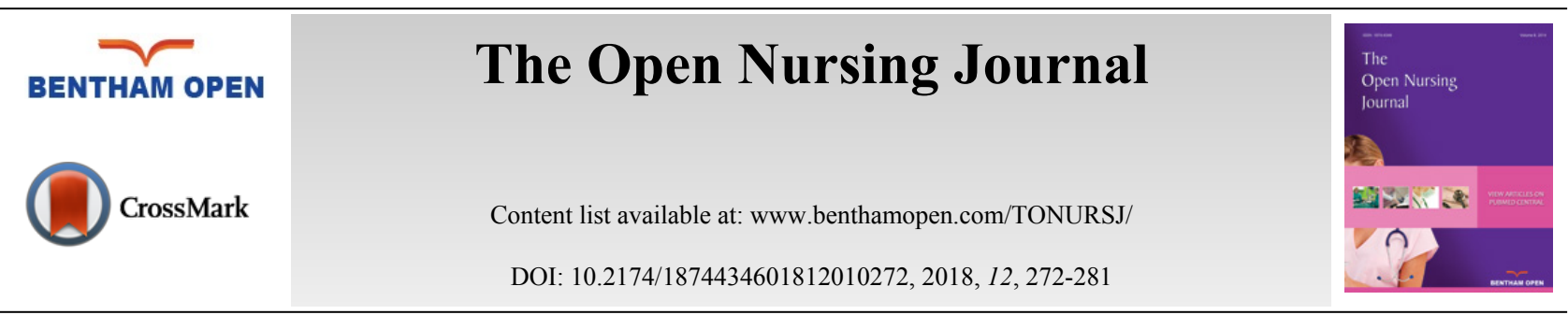

RESEARCH ARTICLE

\title{
An Exploration of Student Midwives' Lived Experiences Regarding Confidence and Satisfaction in Medium-Fidelity Simulation
}

\author{
Zukiswa Brenda Ntlokonkulu*, Ntombana Mcdeline Rala and Daniel Ter Goon \\ Department of Nursing Science, University of Fort Hare, 45 Church Street, East London, Alice, South Africa
}

Received: September 12, 2018

Revised: November 13, 2018

Accepted: November 22, 2018

\section{Abstract:}

Background

Newly qualified midwives are expected to exhibit some level of confidence in practice when they enter the clinical environment.

\section{Objective}

To explore the lived experiences of student midwives after exposure to medium-fidelity simulation concerning confidence and satisfaction.

\section{Methods}

This qualitative, interpretive, phenomenological analysis study was conducted on a purposive sample of five, fourth-year Baccalaureate of Nursing Science student midwives at the University of Fort Hare. In-depth semi-structured interviews were conducted. Data analysis applied the interpretative phenomenological analysis approach.

\section{Results}

Superordinate theme sense of fulfilment elicited six sub-ordinate themes namely 1) Transferability of skills 2) Knowledgeable 3) Equipment used during simulation 4) Realism of simulation 4) Sense of accomplishment 5) Sure/unsure of performance. The use of a standardised patient during the simulation instead of a mannequin prepared participants for the real clinical environment. After being involved in the simulation, participants became more inquisitive regarding the management of the simulated condition. Satisfaction with simulation depended on whether the simulation activity met the student's expectations, and if the simulation equipment resembled real clinical equipment. Post-partum haemorrhage simulation bridged the gap between theory and practice.

\section{Conclusion}

Confidence and satisfaction of student midwives during simulation is depended on the realism of the simulated activity. Exposure to simulated activity increases student knowledge. The student acknowledged that simulation can bridge the gap between theory and practice.

Keywords: Confidence, Interpretative phenomenological analysis, Medium-fidelity simulation, Post-partum haemorrhage, Satisfaction, Standardised patient.

\section{INTRODUCTION}

Limited exposure to patients with variable conditions results in nursing students not receiving the necessary experience to become competent and confident practitioners [1]. This poses a problem in the clinical setting to newly qualified nurses as they are expected to demonstrate certain skills and attributes. Reinhardt et al. [2] assert that for a student to assume a nurse's role, he or she should possess a positive self-disposition. This is attained by focussing on

* Address correspondence to this author at the Department of Nursing Science, University of Fort Hare, 45 Church Street, East London, Alice, South Africa; Tel: +27 43 7047588; Email: zntlokonkulu@ufh.ac.za 
both technical and non-technical skills during simulation, such as critical thinking, confidence and teamwork. It is therefore appropriate that nursing institutions take on the responsibility of implementing teaching strategies that promote learners' confidence and competencies [3].

Confidence during simulation is further influenced by emotions elicited during the simulation experience. Bandura [4] asserts that emotional arousal affects perceived self-belief and abilities when the individual is faced with threatening situations. Simulation can be a catalyst for the student to deal with emotions such as fear, anxiety and stress, which they may encounter in the real clinical environment [5]. Participants from several studies highlighted how simulation helped to reduce anxiety and fear experienced regarding certain procedures in the clinical environment [6, 7], One study, interestingly, also found that simulation can cause students to experience fear and anxiety [8]. These emotions are said to be elicited by exposure to simulation for the first time and being consciously aware of being watched. Finding educational experiences challenging or stressful may, in fact, produce positive changes in learners that may beneficially influence their preconceived assumptions and opinions [9]. Hall [10] affirms that the more students improve their competencies and skills, the more motivated they are to become competent in those skills. Tuzer et al. [11] posit that the development of psychomotor skills is important in the development of nurses' competencies. If students are unable to obtain confidence in skill performance, it becomes impossible to transfer the skill to the clinical environment [2].

Differing simulation fidelities can be used to expedite the transfer of skills to the clinical environment, from task trainers to high-fidelity simulation. Part task trainers, which are low fidelity, are valuable for the attainment of technical skills but do not address the attainment of non-technical skills such as communication [12]. A comparative study conducted by Tuzer et al. [11] on the use of Standardised Patients (SP) and high-fidelity simulators found that HighFidelity Simulation (HFS) enhances transferability of skills. Participants who were exposed to HFS found the mannequin convincing as it had audible lungs and heart compared to the standardised patient SP which could not demonstrate such physiological phenomena. Furthermore, Master of Nursing students in a study by Kowitlawakul et al. [13] found the use of SP restrictive as they were not able to display physiological changes. Experiencing such challenges can interfere with the transferability of skills to the clinical environment.

The creation of an authentic simulation environment is important both for attaining the ultimate simulation experience and for meeting the learning objectives. Several studies have been conducted to evaluate the realism of differing levels of fidelity in [14 - 16]. Participants in these studies perceived simulations that involved standardised patients, community volunteers and hybrid simulators as more realistic than high-fidelity simulators. They considered actors wearing masks more realistic than mannequins [14]. This is in direct contrast to studies that only evaluated the realism of HFS $[17,18]$. The participants in these studies affirmed the realism of the high-fidelity simulation. The participants found the simulation immersive, as the mannequin could speak and had an audible heart and lungs.

In order to be authentic, simulation equipment commonly used in practice needs to be of a kind that students can relate to [19]. This is keeping with Gaba's [20] words that 'simulation is a technique, not a technology' - meaning that emphasis should not be on the equipment itself but on the valid use of the equipment. Argani, et al. [21] state that the biggest mistake that training institutions make is to furnish the simulation laboratory before designing the learning programme. Designing the simulation environment should take into consideration who the learners are and what their learning needs are. The simulation environment should be designed in such a manner that it resembles the real world, from the clock on the wall telling the correct time to the type of documentation used. Studies have demonstrated how seemingly mundane items such as charts and documents can be an important part of a simulation environment [14, 15, 22]. The participants in the study by Luctkar-Flude et al. [15] did not perceive the simulation as real and therefore did not see the importance of the documentation. This contrasts with the findings of another study [14] where 78\% of their participants perceived charts and documents used in simulation as being similar to the clinical environment.

Design of the simulation space is dependent on the cost of the simulation equipment and its availability Gaba, Smith et al. $[20,23]$. state that the cost of the simulation is dependent on the target population, the purpose of the simulation and the technology used. Lapkin et al. [24] conducted a study on the cost-utility analysis of high-fidelity mannequins versus low-fidelity mannequins. The study found that medium-fidelity simulation was cost-effective in helping to impart non-technical skills, such as clinical reasoning, knowledge acquisition and student satisfaction. This is in contrast to the results of a study conducted by Basak et al. [25] comparing HFS and Low-Fidelity Simulation (LFS). The students exposed to LFS scored low in simulation design, concentrating on fidelity amongst other things, compared to the students exposed HFS

There are limited studies that investigate the effect of medium-fidelity simulation on student midwives. Most of the 
literature found is based on High Fidelity Simulation (HFS) using high-fidelity mannequins. Progressive and comparative studies that have been conducted in low, medium and high-fidelity simulation investigated communication, knowledge and clinical readiness [16, 26, 27]. Comparative studies investigating the use of high and low fidelity simulation on student nurses' confidence showed that high-fidelity mannequins increase student confidence more than low-fidelity mannequins [2, 25]. The participants in a study conducted by Fuselier et al. [28] asserted having gained confidence during simulation using mannequins. Furthermore, findings from several studies indicate that HFS enhances confidence in learners [5, 9]. Studies have shown that repeated exposure to HFS increases students' confidence [5]. The aim of the study is to explore the lived experiences of student midwives after exposure to medium-fidelity simulation concerning confidence and satisfaction

\section{METHODS}

An Essential Steps in Management of Obstetric Emergency (ESMOE) post-partum haemorrhage (PPH) video was sent on-line through Blackboard to all fourth-year student midwives in order to demonstrate the process. Management of PPH is one of the contents that are taught in the midwifery high-risk module. The student midwives had an opportunity to watch the video repeatedly in order to thoroughly comprehend the demonstrated skill. The day before simulation the six bedded simulation laboratory was prepared for the PPH simulation. Only four-bed spaces were used for the simulation. The advanced OB Susie a Gaumard simulator with an audible foetal and maternal pulse was filled with simulated blood and urine and was positioned in the centre of the bed. Four large dressing trolleys were prepared with all the equipments needed for the management of PPH. Each bed space had an oxygen outlet with an oxygen mask and two drip stands and a blood pressure machine and relevant ward documentation. The Nursing Science Department recruit members of the community to pretend to be SPs for some of the undergraduate practical program. The SPs were provided with a scenario. Critical points of the scenario were explained to the SPs such as when to call for the nurse, when to pass out and when to regain consciousness, what question to ask. The SPs clad in hospital gown were positioned above the OB Susie torso to create a hybrid. The hybrid was draped with green towels. On the day of the simulation, the students were given the simulation scenario to read prior to the simulation. The ESMOE video was played for the group a second time before the demonstration of the simulation. The students decided among themselves which roles to play during the simulation.

\subsection{Research Design}

An interpretive, phenomenological, analysis (IPA) research design was used in this study to explore, describe and analyse the lived experiences of student midwives regarding the effectiveness of medium-fidelity simulation in an obstetric emergency setting. An interpretative phenomenological analysis research design affords all participants an opportunity to describe and interpret their experiences of MFS [29].

\subsection{Population}

Most of the South African universities offer an undergraduate Bachelor of Nursing Science degree over a period of four years. At the University of Fort Hare midwifery as a module is offered during the last two years of the degree. During the second year of midwifery, abnormal labour including management of obstetric emergencies is taught. The target population was fourth-year Bachelor of Nursing Science student midwives. Student midwives who were the team leaders during the management of postpartum haemorrhage (PPH) using MFS were purposively selected. A small sample size of five participants afforded an in-depth and sufficient information - while not being overly time-consuming [29]. Moreover, a small sample is suggested for the novice IPA researcher [29].

\subsection{Data Collection}

The fourth-year midwifery students were recruited by the first author during their theory block. The first author was a simulation laboratory manager and had no prior relationship with the students. The fourth year group made up of 66 students was approached in class after a lecture. The aim and nature of the study were explained to the students. All 66 students agreed to be part of the study. Out of the eleven student midwives who were team leaders during simulation five were interviewed. All eleven team leaders were given a fair chance to be included, - were entered in the research randomizer software in order to select the five students. The small sample size is deemed adequate to elicit an in-depth response using IPA. The interviews were conducted in the simulation laboratory by the first author. A semi-structured interview guide was used with nine questions with several prompts. Prompts were centred around the student's feelings of the simulation experience. Open-ended questions such as "how did the simulation activity meet your expectations 
regarding confidence in the clinical platform" and "how did you feel thereafter? were used in order to allow the student midwives the opportunity to explore their lived experiences of an obstetric emergency using medium-fidelity simulation. Individual interviews were conducted by the first author in the simulation laboratory. Each interview session lasted between 26 minutes and 44 minutes. A Samsung smartphone was used to record the interviews, and a notepad was used to make notes of gestures such as smiles or other facial expressions. After each interview, the recorded interview was transferred onto a laptop and a file was opened for the interviewee, identified by a pseudonym. The interviews were transcribed verbatim as - Word documents by the first author.

\subsection{Data Analysis}

Data were analysed by the first author following the six steps suggested by Smith et al. [29] namely reading and rereading, initial noting, developing emergent themes, searching for connections across the emergent themes, moving to the next case and looking for patterns across cases. The transcript was read through twice. The first reading was done whilst listening to the recording. During that time any parts of the transcription that did not make sense were clarified by moving back and forth on the recording. During the second reading, the researcher listened in detail, immersed self in the words of the participant. The transcript was analysed line by line, to identify three types of exploratory comments, namely; descriptive, linguistic and conceptual, while trying to make sense of each participant's experiences and being consciously aware not to change original meaning. The researcher tried to interpret the exploratory comments. This is the double hermeneutics of IPA. Then the researcher found a connection between emergent themes. Abstraction was used for the clustering of themes, as each superordinate themes was identified. Some differences were identified in some clusters, indicating polarization. Contextualization was evident in the way participants used their critical thinking and decision-making skills. Each interview was transcribed and analysed separately before moving on to the next case. Finally, all the themes clustered together and commonalities, differences and individuality were identified.

\section{RESULTS}

The super-ordinate theme sense of fulfilment elicited the following subthemes:

\subsection{Transferability of Skill}

Transferability of skill refers to how skills learned during PPH simulation can be applied to the real-world clinical environment: -

The PPH simulation sensitised the participant to the clinical environment. The overall management of PPH practised during simulation, is obviously intended to be transferred to the real clinical environment. The participants became more aware of their clinical environment, and started to ask questions that were relevant to their training, as is evident in the following comments:

'Even now, if you can put me in the real situation clinically at least I'm sure that most of the important things I will be able to do and at the correct time. After that simulation activity, when I arrived in clinical during my allocation, I always make sure that all the equipment is available and the expiry ...' [Lunga]

The exposure to obstetric emergency simulation made the participant feel confident and eager to be involved in a real obstetric emergency. One participant reported:

'I have seen or I have done some of those emergencies, like shoulder dystocia. I'm prepared because our lecture had a specific time to take us to the simulation laboratory and show us how it is done. So I think I am prepared.' [Sino]

Participants reported being immersed in an environment that resembles a real-life situation, thus making it easy for them to apply the acquired knowledge to the real situation. They maintained:

'I think the PPH simulation really helped us to get ready when we go out in the clinical areas, to be able to perform if there are such situations.' [Anga]

'When I go to work next year and be stuck with someone who is having a PPH, at least I will know that somehow I need to insert a condom tamponade to stop the bleeding.' [Zandie]

\subsection{Knowledgeable}

The participants felt that they possessed enough knowledge not only to assist during an emergency but to be actively 
involved in resolving an emergency situation. The student midwives' practical knowledge about PPH increased after the simulation. The participants reported that knowledge of the management of PPH increased during simulation as they were made aware of the steps that are available to manage PPH. This is affirmed in the following quotes:

'... Then I had no idea what's really, really going on but I knew this was a PPH but we were all observing, just getting what was needed. Now being able to do it yourself, then it's a different case, then you like feel more aware.' [Thembi]

'The fact that there are other steps after rubbing the fundus and giving Ringers Lactate, it made you open that you must always try every single option. My knowledge stopped at Ringer's Lactate and of the fundus and bimanual compression.' [Zandie]

The knowledge gained from the simulation made this participant feel confident to go back to the clinical area and implement the knowledge gained:

'When I'm in the ward I will try to inform my colleagues at least to understand their responsibilities during the PPH. They will know how we usually manage the PPH being two people but now at least I'm coming with the idea that let's be four people because I've seen that from the simulation ...' [Lunga]

\subsection{Equipment Used During Simulation}

Concerning the equipment used during simulation, participants maintained that the equipment used was comparable to that used in the clinical environment. To make the simulation as authentic as possible equipment used during management of PPH was provided such as vaginal cuscus, swab holding forceps, artery forceps, green towels, PPH box with contents. They expressed this as follows:

'It is what I expected because I already saw a PPH complication at CMH, [Cecilia Makiwane Hospital]but fortunately, there was a doctor there and they did what we did there, for example, the doctor had a vaginal speculum and she was checking.' [Thembi]

'After that simulation activity when I arrived in the clinical during my allocation I always made sure that all the equipment is available and the expiry date is fine because I always have thoughts of that simulation.' [Lunga]

Participants held that the equipment used during the simulation was high-technology and sophisticated. Some participants saw such advanced high-tech equipment for the first time during the simulation. One participant stressed:

'The equipment used during simulation to me was new, it was at another level. It made me feel like an advanced midwife. When we did a theory on the management of PPH I did not remember hearing anything about using a condom to try and stop the bleeding.' [Zandie]

'Some of the things that were demonstrated or were done, I was not aware of them, for instance, the condom tamponade.' [Sino]

One participant maintained that a well-equipped simulation environment contributed to efficacy:

'The simulation was good, especially all the equipment that was there ...' [Lunga]

\subsection{Realism of Simulation}

Some participants felt that the use of the standardised patient (SP) enhanced the realism of the simulation. The realism of the simulation environment refers to how much it resembled the real world, as is clear from the following comments:

'... if you have a standardised patient that will help the students to be ready during practical, knowing I have faced a real patient so I'm ready.' [Anga]

The participants compared the use of a low fidelity-mannequin with the use of an SP. The interaction that resulted from talking with the patient made the simulation look and feel real, as asserted by the following participant.

'Having the standardised patient was good because Dodo [the mannequin] was not responsive. It's just Dodo she's lying, you're saying whatever you're saying. The SP will talk to you and tell you how she feels compared to Dodo, who just remained quiet.' [Thembi]

The use of the hybrid simulator enhanced the realism of the simulation, as evident in the following comment: 
'We used real patients, yes, even though in some parts we were using the dolls but it's the real human being and the human being is talking. In your mind you know it's not a real situation but you are able to grasp some important points, OK, I missed this.' [Lunga]

The simulation was so realistic that the participants could relate to it as if in a real clinical environment:

'Doing the PPH simulation make you realise that the patient condition can deteriorate. At times the patient's condition does not go the way we learn from the books.' [Zandie]

\subsection{Sense of Accomplishment}

Saving a patient's life and experiencing a generally positive outcome enhances a nurse's sense of accomplishment. Being able to treat the PPH within a reasonable time frame definitely had this effect on the student midwives. Participants said:

'It felt like it's going well after she started regaining consciousness and after the balloon tamponade was stable and not moving at the end, and the vital signs were stable.' [Thembi]

'It depends on the amount of blood loss, whether you did it at the acceptable time. The only thing that we are managing during PPH is to prevent further blood loss. At that time we did manage it properly and the bleeding stopped.' [Anga]

\subsection{Sure or Unsure of Performance}

The participants reported having to stop and think and discuss with team members, prolonging the time taken to resolve the PPH. Being unsure of what to do demanded some discussion, thus using up valuable time. Also, there was lower confidence at the beginning of the simulation, prolonging the time taken to actually deal with the emergency.

'There were times with my group, we will stop and think, "OK what follows after this? What to do next?" Being unsure of what to do actually prolonged the time taken to save the patient.' [Zandie]

'I will say not exactly at the time because it is very rare you will come across management of PPH. It was difficult at first and thus it took us longer to resolve the emergency.' [Sino]

Team members who fail to adhere to their assigned duties may cause some tasks to be executed too late, or not be done at all.

'I can't really, we were not strict with time because the person who was recording time and all the observations was not strict with time. Her job was not just to record observations, as she was also involved in other assignments, so I'm not sure about the time.' [Lunga]

\section{DISCUSSION}

There is mounting evidence that teaching students complex skills in a relaxed, conducive and safe environment increases students' sense of confidence regarding the various skills required in the clinical environment [6]. The use of simulation to teach concepts relevant in midwifery has been widely implemented in order for such skills to be transferred to the clinical environment [30]. The effectiveness of learning transfer during simulation is further stressed by Rush et al. [31].

The use of a standardised patient during the simulation instead of a mannequin has prepared participants for the real clinical environment. This is consistent with the findings of Pike and O'Donnell [32] in their study on the authenticity of the simulation experience, where participants found it difficult to transfer the skills they had acquired after using mannequins. The use of mannequins was reported to have a negative impact on the credibility of the simulation. On the other hand, in a study conducted by Choi [33], student nurses found it difficult to apply the skills they had learned in a psychiatric simulation using a standardised patient. This was attributed to the fact that the real psychiatric patient is not scripted, and therefore will not give the predictable responses and behaviour that the standardised patient gives. A study conducted by Reinhardt et al. [2] supported the use of low fidelity intravenous training arm in teaching students basic clinical skills such as intravenous line insertion. .Lapkin's [24] comparative study found no significant difference between medium and high-fidelity simulation on knowledge acquisition and satisfaction.

The findings of this study showed that after being involved in the simulation, participants became more inquisitive regarding the management of the simulated condition. This is consistent with the findings of the study done by Rush et 
al. [31] on the effect of simulation -- where third-year Bachelor of Nursing students found simulation thoughtprovoking, instigating them to reflect on the care they gave to patients. Similarly, Straub et al. [9] found that an increase in the confidence of students during simulation encouraged them to learn more about obstetric emergencies, which ultimately would improve patient care. Having been exposed to the use of mannequins in the management of obstetric emergencies, one participant in this study felt completely prepared to deal with such emergencies in the clinical environment. This is congruent with the findings of the study done by Brady et al. [16] on the use of varied levels of simulation fidelity, in which students were exposed to progressive-fidelity simulation repeatedly, scoring higher in tests than students exposed only once to one level of simulation fidelity.

The participants in the present study compared their simulation practice with previous experiences of PPH management in a clinical environment. Participants' knowledge of the progressive steps in the management of PPH increased after exposure to PPH simulation. This concurs with Kaplan et al. [34] findings in a study on emergency preparedness disaster simulation (EPDS), where student nurses reported that the use of EPDS increased their knowledge of disaster management. Having acquired new knowledge, one participant in that study became enthusiastic about sharing his knowledge in the clinical environment with other staff members. Reinhardt et al. [2] asserted that success in the clinical environment was influenced by the student's level of confidence in the simulation environment.

The findings of this study showed that participants' satisfaction with simulation depended on whether the simulation activity met the student's expectations, and if the simulation equipment resembled real clinical equipment. Smith et al. [23] stressed that the simulation environment should reflect reality as much as possible. Simulation attributes enable learning in undergraduate students [35]. The third-year midwifery students in a study conducted by Carolan-Olah et al. [36] expressed low confidence in using the resuscitation equipment. This was attributed to the fact that the student midwives found the simulation stressful, causing them to focus more on simulating the scenario than on using the equipment correctly.

The participants in this study said that the simulation enabled them to see and use some of the equipment for the first time. The participants found the experience overwhelming, but this did not deter their participation. In Kaplan et al.'s [34] study o students found the equipment realistic, shocking and overwhelming. The students in that study found the simulation more effective and realistic than others they had experienced because of the high standard of equipment used. Brady et al. [16] posit that inadequate equipment hinders simulation learning. In the present study, the participants were satisfied with the simulation because it was well-equipped - enhancing experiential learning

The realism of the simulation environment is the key determinant to how effective the simulation is. The simulation experience must be immersive enough to make the student become 'lost in the moment'. The use of the standardised patient was seen by one participant as adequate preparation for the real clinical environment. These findings are consistent with the findings of Choi [33] on the use of standardised patients during psychiatry simulation which made the student imagine what the clinical environment would look like.

Comparing the SP with the use of a low-fidelity mannequin made participants aware of the benefits associated with the use of the SP. The fact that the SP was able to communicate helped students to understand the situation. This is comparable with the findings of Kaplan et al. [34] on the incorporation of real patients during emergency preparedness disaster simulation. The students in that study reported that increased realism help them to immerse themselves in the situation and respond more accurately. The synergy between the two simulation models, namely the task trainer and the $\mathrm{SP}$, increased the realism of the simulation [23, 37]. This is consistent with the findings of this study, as one of the participants acknowledged the use of the SP and part-task trainer. The participant reported how communicating with the SP made the simulation authentic.

Participants identified the gap between theory and practice and how the PPH simulation had bridged that gap. Participants felt that the PPH simulation painted a clear picture of how things can rapidly deteriorate during real-life situations. Yardley et al. [38] suggest that the learning gaps that are identified in teaching modalities should not be used to find faults but to point to areas to focus on in practice. Simulation is the ideal tool to bridge the gap between theory and practice [18].

Successfully resolving PPH does not depend only on the time that elapses from the onset of bleeding, but also on factors such as the amount of blood loss, the cause and origin of the PPH and general maternal condition. One participant verbalised that they wasted valuable time discussing options among themselves. However, two participants felt that they had resolved the PPH within an acceptable time and that discussion had helped them to locate the cause of the bleeding. Kurrek et al. [39] posit that in a PPH emergency, time may not be an optimal indicator of performance and 
competence. They further argue that a set time may not work well when there are many possibilities to resolve the problem. Su [40] assert that clinicians who are caring for a pregnant woman should be skilled and knowledgeable to manage patients with PPH timeously. They further conclude that there should be a dedicated person to record the sequence of events and the timing of medical interventions.

\section{LIMITATIONS}

The study focused on the lived experiences of fourth-year student midwives utilising simulation laboratories at one university, its findings cannot be generalized to other universities in South Africa. Therefore, exploring the views of the nursing students in other universities is needed.

\section{CONCLUSION}

The ultimate desired outcome of simulated experience is to increase the student confidence thus making them competent clinical practitioners. The participants were able to relate the simulation to the real clinical environment. Simulation increased the student confidence in making them feel that they can at least try to manage an obstetric emergency when confronted by it. The participant knowledge of the management of PPH was increased after exposure to the simulation.

\section{ETHICS APPROVAL AND CONSENT TO PARTICIPATE}

Ethical approval was obtained from the University of Fort Hare ethics committee.

\section{HUMAN AND ANIMAL RIGHTS}

No animals/humans were used for studies that are the basis of this research.

\section{CONSENT FOR PUBLICATION}

Participants were made to understand their right to privacy of any information provided, and their consent was sought before the use of a tape recorder. Prior to the interview, each participant signed an informed consent form. Before the beginning of each interview, permission was sought from each participant to use a pseudonym.

\section{CONFLICT OF INTEREST}

None declared.

\section{ACKNOWLEDGEMENTS}

The authors would like to thank the student midwives who took part in this study.

ZN designed the study, collected data, analysed and wrote the first draft. NR and DG provided inputs in the data analysis and writing up of the paper. All authors critically revised and approved the manuscript.

\section{REFERENCES}

[1] Aggarwal R, Mytton OT, Derbrew M, et al. Training and simulation for patient safety. Qual Saf Health Care 2010; 19(Suppl. 2): i34-43. [http://dx.doi.org/10.1136/qshc.2009.038562] [PMID: 20693215]

[2] Reinhardt AC, Mullins IL, De Blieck C, Schultz P. IV insertion simulation : Confidence, skill, and performance. Clin Simul Nurs 2012; 8(5): e157-67. [Internet].

[http://dx.doi.org/10.1016/j.ecns.2010.09.001]

[3] Yuan HB, Williams BA, Fang JB. The contribution of high-fidelity simulation to nursing students ' confidence and competence : A systematic review. Int Nurs Rev 2012; (59): 26-33. [http://dx.doi.org/10.1111/j.1466-7657.2011.00964.x]

[4] Bandura A. Self-efficacy: Toward a unifying theory of behavioral change. Psychol Rev 1977; 84(2): 191-215. [http://dx.doi.org/10.1037/0033-295X.84.2.191] [PMID: 847061]

[5] Kaddoura M, Vandyke O, Smallwood C, Gonzalez KM. Perceived benefits and challenges of repeated exposure to high fidelity simulation experiences of first degree accelerated bachelor nursing students. Nurse Educ Today 2016; 36: 298-303. [http://dx.doi.org/10.1016/j.nedt.2015.07.014] [PMID: 26260522]

[6] Partin JL, Payne TA, Slemmons MF. Students' perceptions of their learning experiences using high-fidelity simulation to teach concepts relative to obstetrics. Nurs Educ Perspect 2011; 32(3): 186-8. [http://dx.doi.org/10.5480/1536-5026-32.3.186] [PMID: 21834381] 
[7] Reid-Searl K, Happell B, Vieth L, Eaton A. High fidelity patient silicone simulation: A qualitative evaluation of nursing students' experiences. Collegian 2012; 19(2): 77-83. [http://dx.doi.org/10.1016/j.colegn.2011.09.003] [PMID: 22774349]

[8] Pollock C, Biles J. Discovering the lived experience of students learning in immersive simulation. Clin Simul Nurs 2016; 12 : 313-9. [http://dx.doi.org/10.1016/j.ecns.2016.03.002]

[9] Straub HL, Morgan G, Ochoa P, et al. Targeted obstetric haemorrhage programme improves incoming resident confidence and knowledge. J Obstet Gynaecol 2013; 33(8): 798-801. [http://dx.doi.org/10.3109/01443615.2013.816668] [PMID: 24219716]

[10] Hall SW. High-Fidelity Simulation for Senior Maternity Nursing Students. Nurs Educ Perspect 2015; 36(2): $124-7$. [http://dx.doi.org/10.5480/12-996.1] [PMID: 29194140]

[11] Tuzer H, Dinc L, Elcin M. The effects of using high-fidelity simulators and standardized patients on the thorax, lung, and cardiac examination skills of undergraduate nursing students. Nurse Educ Today 2016; 45: 120-5. [http://dx.doi.org/10.1016/j.nedt.2016.07.002] [PMID: 27449150]

[12] Kneebone R, Nestel D, Wetzel C, et al. The human face of simulation: patient-focused simulation training. Acad Med 2006; 81(10): 919-24. [http://dx.doi.org/10.1097/01.ACM.0000238323.73623.c2] [PMID: 16985358]

[13] Kowitlawakul Y, Chow YL, Salam ZHA, Ignacio J. Exploring the use of standardized patients for simulation-based learning in preparing advanced practice nurses. Nurse Educ Today 2015; 35(7): 894-9. [http://dx.doi.org/10.1016/j.nedt.2015.03.004] [PMID: 25819268]

[14] Kable AK, Arthur C, Levett-Jones T, Reid-Searl K. Student evaluation of simulation in undergraduate nursing programs in Australia using quality indicators. Nurs Health Sci 2013; 15(2): 235-43. [http://dx.doi.org/10.1111/nhs.12025] [PMID: 23240779]

[15] Luctkar-Flude M, Wilson-Keates B, Larocque M. Evaluating high-fidelity human simulators and standardized patients in an undergraduate nursing health assessment course. Nurse Educ Today 2012; 32(4): 448-52. [http://dx.doi.org/10.1016/j.nedt.2011.04.011] [PMID: 21565436]

[16] Brady S, Bogossian F, Gibbons K. The effectiveness of varied levels of simulation fidelity on integrated performance of technical skills in midwifery students - A randomised intervention trial. Nurs Educ today 2015; 35: 524-9.

[17] Maas NA, Flood LS. Implementing high-fidelity simulation in practical nursing education. Clin Simul Nurs 2011; 7(6): e229-35. [Internet] [http://dx.doi.org/10.1016/j.ecns.2010.04.001]

[18] Thidemann IJ, Söderhamn O. High-fidelity simulation among bachelor students in simulation groups and use of different roles. Nurse Educ Today 2013; 33(12): 1599-604. [http://dx.doi.org/10.1016/j.nedt.2012.12.004] [PMID: 23302256]

[19] Roh YS, Lee WS, Chung HS, Park YM. The effects of simulation-based resuscitation training on nurses' self-efficacy and satisfaction. Nurse Educ Today 2013; 33(2): 123-8

[http://dx.doi.org/10.1016/j.nedt.2011.11.008] [PMID: 22153054]

[20] Gaba DM. The future vision of simulation in health care. Qual Saf Health Care 2004; 13(Suppl. 1): i2-i10. [http://dx.doi.org/10.1136/qshc.2004.009878] [PMID: 15465951]

[21] Argani CH, Eichelberger M, Deering S, Satin AJ. The case for simulation as part of a comprehensive patient safety program. Am J Obstet Gynecol 2012; 206(6): 451-5.

[http://dx.doi.org/10.1016/j.ajog.2011.09.012] [PMID: 22000670]

[22] Levett-Jones T, Andersen P, Reid-Searl K, et al. Tag team simulation: An innovative approach for promoting active engagement of participants and observers during group simulations. Nurse Educ Pract 2015; 15(5): 345-52. [http://dx.doi.org/10.1016/j.nepr.2015.03.014] [PMID: 25936431]

[23] Smith A, Siassakos D, Crofts J, Draycott T. Simulation: Improving patient outcomes. Semin Perinatol 2013; 37(3): 151-6. [http://dx.doi.org/10.1053/j.semperi.2013.02.005] [PMID: 23721770]

[24] Lapkin S, Levett-Jones T. A cost-utility analysis of medium vs. high-fidelity human patient simulation manikins in nursing education. J Clin Nurs 2011; 20(23-24): 3543-52. [http://dx.doi.org/10.1111/j.1365-2702.2011.03843.x] [PMID: 21917033]

[25] Basak T, Unver V, Moss J, Watts P, Gaioso V. Beginning and advanced students' perceptions of the use of low- and high-fidelity mannequins in nursing simulation. Nurse Educ Today 2016; 36: 37-43. [http://dx.doi.org/10.1016/j.nedt.2015.07.020] [PMID: 26282193]

[26] McCaughey CS, Traynor MK. The role of simulation in nurse education. Nurse Educ Today 2010; 30(8): 827-32. [http://dx.doi.org/10.1016/j.nedt.2010.03.005] [PMID: 20483188]

[27] Lapkin S, Fernandez R, Levett-Jones T, Bellchambers H. The effectiveness of using human patient simulation manikins in the teaching of clinical reasoning skills to undergraduate nursing students: a systematic review. Clin Simul Nurs 2010; 8(16): 661-94. [http://dx.doi.org/10.1016/j.ecns.2010.05.005] [PMID: 27820553]

[28] Fuselier J, Baldwin D, Townsend-Chambers C. Nursing Students' Perspectives on manikins of colour in simulation laboratories. Clin Simul 
Nurs 2016; 12(6): 197-201.

[http://dx.doi.org/10.1016/j.ecns.2016.01.011]

[29] Smith JA, Flowers P, Larkin M. Interpretative phenomenological analysis theory, method and research. London: Sage Publications Ltd 2009.

[30] Reynolds A, Ayres-de-Campos D, Lobo M. Self-perceived impact of simulation-based training on the management of real-life obstetrical emergencies. Eur J Obstet Gynecol Reprod Biol 2011; 159(1): 72-6. [http://dx.doi.org/10.1016/j.ejogrb.2011.07.022] [PMID: 21831504]

[31] Rush S, Acton L, Tolley K, Marks-Maran D, Burke L. Using simulation in a vocational programme:does the method support the theory? J Vocat Educ Train 2010; 62(4): 467-79.

[http://dx.doi.org/10.1080/13636820.2010.523478]

[32] Pike T, O'Donnell V. The impact of clinical simulation on learner self-efficacy in pre-registration nursing education. Nurse Educ Today 2010; 30(5): 405-10.

[http://dx.doi.org/10.1016/j.nedt.2009.09.013] [PMID: 19883960]

[33] Choi YJ. Exploring experiences of psychiatric nursing simulations using standardized patients for undergraduate students. Asian Nurs Res 2012; 6(3): 91-5.

[http://dx.doi.org/10.1016/j.anr.2012.07.001] [PMID: 25030974]

[34] Kaplan BG, Connor A, Ferranti EP, Holmes L, Spencer L. Use of an emergency preparedness disaster simulation with undergraduate nursing students. Public Health Nurs 2012; 29(1): 44-51.

[http://dx.doi.org/10.1111/j.1525-1446.2011.00960.x] [PMID: 22211751]

[35] Bland AJ, Tobbell J. Towards an understanding of the attributes of simulation that enable learning in undergraduate nurse education : A grounded theory study. Nurse Educ Today 2016; 44: 8-13. [http://dx.doi.org/10.1016/j.nedt.2016.05.011]

[36] Carolan-Olah M, Kruger G, Brown V, Lawton F, Mazzarino M. Development and evaluation of a simulation exercise to prepare midwifery students for neonatal resuscitation. Nurse Educ Today 2016; 36: 375-80.

[http://dx.doi.org/10.1016/j.nedt.2015.09.009]

[37] Siassakos D, Draycott T, O’Brien K, Kenyon C, Bartlett C, Fox R. Exploratory randomized controlled trial of hybrid obstetric simulation training for undergraduate students. Simul Healthc 2010; 5(4): 193-8.

[http://dx.doi.org/10.1097/SIH.0b013e3181d3ee0b] [PMID: 21330796]

[38] Yardley S, Irvine AW, Lefroy J. Minding the gap between communication skills simulation and authentic experience. Med Educ 2013; 47(5): 495-510. [http://dx.doi.org/10.1111/medu.12146] [PMID: 23574062]

[39] Kurrek MM, Morgan P, Howard S, Kranke P. Simulation as a new tool to establish benchmark outcome measures in obstetrics. PLoS One 2015; 10(6): 1-10.

[40] Su LL, Chong YS. Massive obstetric haemorrhage with disseminated intravascular coagulopathy. Best Pract Res Clin Obstet Gynaecol 2012; 26(1): 77-90.

[http://dx.doi.org/10.1016/j.bpobgyn.2011.10.008] [PMID: 22101177]

(C) 2018 Ntlokonkulu et al.

This is an open access article distributed under the terms of the Creative Commons Attribution 4.0 International Public License (CC-BY 4.0), a copy of which is available at: (https://creativecommons.org/licenses/by/4.0/legalcode). This license permits unrestricted use, distribution, and reproduction in any medium, provided the original author and source are credited. 